\title{
Remote Interviews and Visual Timelines with Patients: Lessons Learned
}

\author{
Paula VALKONEN ${ }^{\mathrm{a}, 1}$, Nina KARISALMI ${ }^{\mathrm{a}}$, Johanna KAIPIO ${ }^{\mathrm{a}}$ and Sari KUJALA ${ }^{\mathrm{a}}$ \\ a Aalto University, Department of Computer Science, Espoo, Finland
}

\begin{abstract}
This article aims to support the design of remote user studies in the healthcare and well-being field. We introduce lessons learned from conducting remote interviews and using visual timelines as pretasks involving patients from two eHealth projects. Based on our experience, we conclude that remote interviews and visual timelines can provide rich data about user needs. However, careful planning is required. Building trust, rapport, confidentiality, and privacy requires extra effort from the researcher in studies involving sensitive topics. In this paper, we present a list of practical tips for planning qualitative health-related user studies.
\end{abstract}

Keywords. eHealth, patients, remote interviews, user needs, visual timeline

\section{Introduction}

In user research, interviews are used to gather qualitative data about people's views, experiences, and motivations [1]. Advancements in technology have enabled researchers to develop new ways of conducting interviews; however, in-person interviews are often considered better than remote options regarding the quality of data [2].

Recently, it has been argued that remote interviews may offer some advantages [2]. Skype interviews have been shown to be popular [3], as this mode is a practical and costeffective way of interviewing people globally without the need to travel [4]. In addition, remote interviews make rescheduling easier $[3,4]$ and can be utilized even when a participant has difficult times [4]. Studies have not shown a significant difference between in-person, telephone, and Skype interviews regarding data quality [2,3]. Privacy and the interview context may be more important in generating high-quality data than the chosen technique [3].

Creative methods such as drawing tasks can be used in connection with interviews to support the participants in reflecting on the issue more broadly and in new ways [5]. Even though the benefits outweigh the barriers, the researcher needs to consider the participants' possible fears, especially regarding tasks that require skills [5]. In face-toface interviews, the co-creation of customer journey maps and visual timelines can be done during the interview to support the discussion between the participant and the researcher [6]. However, in remote interviews, the practice of similar activities requires special consideration and arrangements [7].

Even though several research guides have explained the interview method, no study has explored remote research practically from different perspectives to the best of our

${ }^{1}$ Corresponding Author, Paula Valkonen, Department of Computer Science, PO Box 15400, FI-00076 AALTO, Finland, Email: paula.valkonen@aalto.fi. 
knowledge. This article aims to support the design of remote user studies in the healthcare and well-being field, especially research that deals with sensitive and personal topics where data security issues and management require special attention. In this paper, we describe experiences from two studies where we applied, due to the COVID-19 pandemic, remote user research methods, particularly interviews and probes, to study the experiences and needs of patients as end users of eHealth solutions. In the context of this paper, remote interview techniques include phone interviews and videoconference techniques using Skype or Teams. The research questions were as follows:

- Compared to a face-to-face setup, which issues require special attention when conducting remote user research with patients?

- What kinds of practical arrangements can be used to support privacy, trust, rapport, and confidentiality in remote user research?

\section{Methods}

The research was carried out in two ongoing projects: DigiIN and eHealth in Home Dialysis (Table 1). In both research projects, the focus was on patients' everyday lives, related experiences and needs, and the use of eHealth services.

Table 1. Description of the studies in two research projects

\begin{tabular}{|c|c|c|}
\hline & DigiIN & eHealth in Home Dialysis \\
\hline Focus & $\begin{array}{l}\text { Patient needs for eHealth services; } \\
\text { feedback on a specific eHealth } \\
\text { service }\end{array}$ & $\begin{array}{l}\text { Experiences and needs of patients with } \\
\text { kidney disease; participatory development } \\
\text { of eHealth solutions for home dialysis }\end{array}$ \\
\hline Duration & 2019-2025 & $2020-2021$ \\
\hline $\begin{array}{l}\text { Remote user } \\
\text { research methods }\end{array}$ & $\begin{array}{l}\text { Remote interviews, probes (incl. a } \\
\text { visual timeline task) }\end{array}$ & $\begin{array}{l}\text { Remote interviews, paper-based visual } \\
\text { timeline pretask }\end{array}$ \\
\hline Participants & Thirty older adults & $\begin{array}{l}\text { Thirty patients with kidney disease and } \\
\text { eight healthcare personnel (nurses and } \\
\text { physicians) }\end{array}$ \\
\hline Recruitment & $\begin{array}{l}\text { With the help of healthcare } \\
\text { personnel and a web questionnaire }\end{array}$ & $\begin{array}{l}\text { With the help of healthcare personnel as a } \\
\text { part of a larger project }\end{array}$ \\
\hline $\begin{array}{l}\text { Remote interview } \\
\text { technology }\end{array}$ & $\begin{array}{l}\text { Teams, with video connection } \\
\text { when a participant requested for it }\end{array}$ & $\begin{array}{l}\text { Teams: video call primarily, audio call } \\
\text { when participants requested for it }\end{array}$ \\
\hline $\begin{array}{l}\text { Remote interview } \\
\text { duration }\end{array}$ & $1 \mathrm{~h}$ and $2 \times 15 \mathrm{mins}$ & $\begin{array}{l}1 \mathrm{~h} \text { for healthcare personnel; } 2 \mathrm{~h} \text { for } \\
\text { patients }\end{array}$ \\
\hline
\end{tabular}

In DigilN, the needs of different user groups (including the potentially most vulnerable groups, such as older persons and socially excluded groups) were investigated. In this study, we focused on older adults who had some well-being-related challenges that they desired to work on as part of the study. First, the participants were briefed about the study via phone call. Next, participants were asked to complete a probe [8] with a visual timeline task [9]. The probe aimed at collecting user needs indirectly by thoroughly exploring the respondents' healthy habits. The time allowed to fill out the probe was two to four weeks, during which the researcher called the participants twice. On both occasions, the participants had the opportunity to ask questions about the study. Short interviews were also conducted, and the calls were recorded. The calls helped to engage the participants, increased rapport, and prepared them for the final phase of the study - the remote interview [7]. Data used in this article include experiences from 14 interviews with participants between 61 and 90 years. 
In eHealth in Home Dialysis, the research aimed to support the development of eHealth solutions for patients with kidney disease from the viewpoint of user needs, usability, and user experience. In the first phase of the study, the participants were briefed about the study via phone and had the opportunity to ask questions. Next, the participants were provided with a paper-based assignment in which professionals were asked to describe the course of treatment on a visual timeline [9]. Similarly, the patients were asked to describe their treatment paths by adding information and descriptions to the timeline and expressing their moods and feelings with color stickers. They were also asked to identify the most memorable incident during the timeline period. The pretask aimed to collect information on the participants' earlier treatment situations. Visual timelines as pretasks helped the researchers to understand the patients' experiences of the treatment over a long period. The remote interview was conducted about one week after the participants completed the pretask. Data used in this article include experiences from six interviews with patients aged 30-60 years old and six patients over 60 years old, as well as eight interviews with healthcare professionals.

\section{Results: Lessons learned}

Based on experiences from two eHealth studies, the study arrangements worked well, and the methods used provided rich data about user needs. Even though the studies were conducted remotely, the participants found the research pleasant and acceptable. We identified the following key learnings which we present as our results.

In remote interviews, careful planning is important. Compared to face-to-face sessions, remote interviews, including interviews about sensitive and personal topics, require more careful planning, especially from a security and data protection perspective. The technology platform, including a safe recording system and secure scanning options, security in all aspects of the data flow, and participants' privacy must be carefully considered. The technical arrangement, including the phone number the researcher used to call, was explained to the nontechnical participants to gain their trust. A contingency plan was also communicated in case of technology failure. Moreover, careful planning minimized the risks of uncontrolled partitions: repeated reminders of key research actions, optimization of paper-based pretask and consent form logistics via traditional mail, and organizing enough flexible time in the research schedule helped us to conduct the research in changing situations.

Pretasks improved the participants' orientation regarding the interview but brought up challenges with utilizing the task deliverables during the remote sessions. Compared to a face-to-face interview, co-creation of the task, such as drawing a timeline in a remote interview, was challenging since it required a video connection, the use of specific digital tools, skills from the participants, as well as extra time. To overcome these challenges, we asked the participants to fill out the paper-based timeline before the interview. In the eHealth in Home Dialysis project, the participants mailed the filled timeline papers to the researchers before the interview, who then digitized and shared the illustrations via a screen during the discussion. This procedure enabled the researchers to prepare timelinespecific questions for the interview and to discuss the illustration with the participants while they both looked at it together. However, mailing the paper-based pretask back and forth was time-consuming. In contrast, in the DigiIN project, the participants were requested to mail paper-based probes, including visual timelines, to the researcher after the interview. This arrangement made the interview schedule more flexible, although the 
researcher could not see the visualization during the interview nor prepare related questions. In the interviews, the prefilled probe with the older adults supported their memory. All in all, the visual timeline pretask was found to work well in both projects as part of remote interviews: the sessions proceeded logically and the participants could think about the content adequately (e.g., checking special dates from calendars). The creative tasks were appreciated, although, a few of the participants found the timelines difficult to fill in. In face-to-face interviews, participants usually have less time to think and organize their thoughts. From this perspective, in the remote interviews with the pretasks, the participants had more empowerment than without them.

Building trust and rapport remotely requires extra effort from the researchers. In remote interviews, especially without video connections, researchers must trust their listening skills, as it is difficult to know the mood of the participant. We found that when building trust and creating rapport for remote interviews, including interviews on sensitive topics, the first contact with the participant is vital. With this in mind, the researcher acting as an interviewer made the first introductory phone call to the participant. This arrangement enabled the participant to save the researcher's phone number for further calls. The chat between the participant and the researcher before the actual remote interview was helpful in discussing sensitive topics, thoroughly exploring the questions productively during the interview, and creating an open atmosphere during the interviews.

Confidentiality and privacy must be considered during remote sessions and when managing and transferring sensitive data. Although many patients described their situations and experiences quite openly in health and well-being studies, it is important to maintain confidentiality and privacy in all phases of the study and inform the participants of this. For example, we used a local scanner instead of a shared scanner connected to the cloud service to scan the consent forms and headsets during the interview in a private location to prevent outsiders from hearing the conversation.

\section{Discussion and conclusions}

This paper describes our experiences from two health and wellbeing-related user studies. Due to COVID-19, we utilized remote user research methods, particularly interviews and visual timelines. Still, our experiences revealed that remote studies require more careful planning compared to face-to-face interviews, and there is a need to revise the methods for the remote setup. We found that pretasks, including visual timelines, supported remote interviews. The most obvious difference between in-person and remote setups seemed to be related to building trust, which is more challenging during remote interviews. We present a list of practical tips that can be utilized when planning qualitative health and wellbeing-related user studies:

- To build trust, contact the participant multiple times. Preferably, this should be done by the researcher who will conduct the interview.

- Identify the secure remote tool and technologies your organization provides for the research and allow the participants to choose the one they prefer (e.g., phone or video conferencing tools). Acknowledge that participants have different technology skills. Also, let them know that their video cameras can be turned off.

- Utilize creative pretasks in the study to efficiently explore the interview topics. 
- Ensure the fluent use of technical equipment for both the participant and the researcher (including devices, software, surroundings, etc.).

- Make sure data transfer is secure at all levels-get familiar with the cloud services where the data may be stored and do not allow the transfer of data to countries outside the privacy notice.

- Be prepared for various kinds of technical problems and surprises. When multiple interviewers are involved, delegate tasks beforehand to them.

In studies dealing with sensitive health-related topics, trust, ethical considerations, privacy, and information security are essential. In remote user studies, these issues are prominent since participants' technology literacy may vary, and they rely on things they cannot directly observe themselves.

Based on our experience, it is possible to conduct remote interviews, just like faceto-face sessions. We collected high-quality data despite the remote arrangements. However, the lack of nonverbal clues hampered the building of trust and rapport during the interviews, and this should be further studied.

\section{Acknowledgments and ethics approval}

DigilN was supported by the Strategic Research Council at the Academy of Finland [numbers 327145 and 327147]. The project eHealth in Home Dialysis was part of CleverHealth Network ecosystem and the project (eCareForMe Homedialysis) was funded by participating companies, Hospital District of Helsinki and Uusimaa, and Business Finland. We thank our project partners, colleagues Sini Leinonen and Sini Hölsä from Aalto University, Virpi Rauta and Tiina Puurtinen and Pirgit Silvast-Äikäs from Helsinki University Hospital, and from Terveystalo Riina-Riitta Helminen, Iina Vartia, Reija Saarinen and Sanna Solala. eHealth in Home Dialysis received permission from the ethical committee of the Hospital District of Helsinki and Uusimaa (HUS/1649/2020, Jarkko Ihalainen), while DigiIN received permission from the AU Ethics Committee (95_03.04_2019_DigiIN, Ossi Naukkarinen).

\section{References}

[1] Gill P, Stewart K, Treasure E, Chadwick B. Methods of data collection in qualitative research: interviews and focus groups. British dental journal. 2008 Mar;204(6):291-5.

[2] Johnson DR, Scheitle CP, Ecklund EH. Beyond the In-Person Interview? How Interview Quality Varies Across In-person, Telephone, and Skype Interviews. Soc. Sci. Comput. Rev. 2019 Dec 11:1-11.

[3] Jenner BM, Myers KC. Intimacy, rapport, and exceptional disclosure: a comparison of in-person and mediated interview contexts. Int. J. Soc. Res. Methodol. 2019 Mar 4;22(2):165-77.

[4] Brown N. Video-conference interviews: Ethical and methodological concerns in the context of health research. SAGE Research Methods. 2018.

[5] Rainford J. Confidence and the effectiveness of creative methods in qualitative interviews with adults. Int. J. Soc. Res. 2020 Jan 2;23(1):109-22.

[6] Bowen S, Durrant A, Nissen B, Bowers J, Wright P. The value of designers' creative practice within complex collaborations. Des Stud. 2016 Sep 1;46:174-98.

[7] Hillman S, Forghani A, Pang C, Neustaeder C, Judge TK. Conducting interviews with remote participants. Studying and Designing Technology for Domestic Life. 2014 Sep 26:11-53.

[8] Gaver B, Dunne T, Pacenti E. Design: cultural probes. interactions. 1999 Jan 1;6(1):21-9.

[9] Pell B, Williams D, Phillips R, Sanders J, Edwards A, Choy E, Grant A. Using Visual Timelines in Telephone Interviews: Reflections and Lessons Learned From the Star Family Study. IJQM. 2020 Mar 27;19:1-11. 